\title{
Expresiones de afecto de madres bilingües ${ }^{1}$
}

\author{
Bilingual mothers' expressions of affect
}

\author{
Martha Shiro ${ }^{2}$ \\ Universidad Central de Venezuela, Venezuela
}

Resumen. En la interacción de 10 madres bilingües con sus hijos de 30 meses se analiza la expresión de afectividad en L1 y en L2. Se identificaron las expresiones de emoción, volición y actitud epistémica en 30 interacciones espontáneas: (a) 10 madres (español L1) hablando en L1, (b) 10 madres (español L1) hablando en inglés L2 y (c) 10 madres (inglés L1) hablando en L1. Los resultados sugieren que los usos de L2 difieren de los de L1 (inglés o español): la expresión de volición y actitud epistémica se asemeja al inglés L1, mientras que la expresión de las emociones se acerca al español L1. Estos hallazgos permiten explicar los usos del lenguaje expresivo de los bilingües y ayudan a determinar sus efectos en el desarrollo del lenguaje.

Palabras clave: afectividad, input materno, bilingüismo, desarrollo de lenguaje.

Abstract. The purpose of this study is to examine the uses of affectivity in L1 and L2 in 10 bilingual mothers' interactions with their 30-month olds. The expressions of emotion, volition and epistemic stance were identified in 30 spontaneous mother-child interactions: (a) 10 Spanish L1 mothers speaking in L1; (b) 10 Spanish L1 mothers speaking in English L2; and (c) 10 English L1 mothers speaking in L1. The results suggest that L2 uses differ from L1: expressions of volition and epistemic stance in English L2 resemble their use in English L1, whereas expressions of emotions in English L2 are similar to Spanish L1. These findings shed light on bilingual speakers' expressive language and are useful in determining the impact on language development.

Key words: Affectivity, maternal input, bilingualism, language development.

\footnotetext{
${ }^{1}$ Gracias a Erika Hoff y a su equipo de investigación por el constante apoyo para que este trabajo se realizara. Agradezco, en particular, la valiosa ayuda de KatFilippi en las diferentes etapas de este estudio y a Javier Ponce, por colaborar en la codificación de los datos. Los datos de esta investigación provienen de un proyecto de la Dra. Erika Hoff financiadopor The Eunice Kennedy Shriver National Institute of Child Health and Human Development, HD068421.

${ }^{2}$ Martha Shiro, Instituto de Filología “Andrés Bello“, Universidad Central de Venezuela. E-mail: shiromartha@gmail.com Dirección postal: Apartado 47141 Los Chaguaramos Caracas 1041A Venezuela.
} 


\section{Introducción}

En este trabajo se comparan las expresiones de afectividad que usan las madres bilingües nativas del español cuando interactúan con sus hijos tanto en español como en inglés, en situaciones similares. El objetivo del estudio es determinar si las expresiones de afectividad varían en hablantes bilingües cuando se comunican en una o en otra lengua y si el uso que hacen de las expresiones afectivas se asemeja al uso de los hablantes nativos. Este estudio forma parte de un proyecto mayor cuyo propósito es determinar el impacto de estas variaciones en las expresiones de afectividad de los niños expuestos a un entorno bilingüe y comprobar si estas habilidades expresivas se incorporan posteriormente a las habilidades discursivas, particularmente a la construcción de la perspectiva narrativa (Shiro, 2003).

Las investigaciones que examinan la interacción temprana reportan que las normas culturales rigen el desarrollo de los intercambios y tienen un papel fundamental en la socialización de los niños (Ochs, 1988; Schiefflin \& Ochs, 1986; Chen et al. 2012). Para reflexionar sobre cómo se incorporan las normas culturales en el habla infantil es necesario examinar los usos de la lengua en los que están inmersos los niños desde que nacen, puesto que el habla que la madre dirige al niño afecta el desarrollo del lenguaje infantil (Hoff, 2013). En particular, según Eisenberg et al. (1998), la expresividad en el lenguaje de los padres puede influir en las habilidades de los niños para interpretar y comprender las reacciones emocionales de los demás. Las expresiones de emoción suministran información acerca del significado de los eventos, las conductas que los acompañan y la reacción de los demás a las emociones. Las expresiones de emotividad de los padres también sirven para exponer al niño a una gama de emociones. Se piensa que la habilidad de interpretar las emociones de los demás se relaciona con la facilidad con la cual los niños interactúan con otros, de modo que se presume que las expresiones de emociones de los padres influyen en la comprensión de las emociones de los hijos y, de esta manera, contribuyen a sus habilidades sociales.

Desde una perspectiva funcionalista, como la que se adopta en este trabajo (Halliday, 1994), se parte del supuesto de que los múltiples usos de la lengua delimitan las formas lingüísticas, según los propósitos comunicativos de los participantes en la interacción. El significado, entonces, se construye en varios planos: el experiencial o ideacional-que nombra, describe, hace referencia y aprehende al mundo-, el interpersonal -que construye el vínculo entre los participantes-, y el textual -que teje los lazos entre los enunciados para construir el discurso-.

El foco central de esta investigación es la expresión de la afectividad que se ubica en el plano interpersonal y se le conoce también como función expresiva o evaluativa del lenguaje, en contraposición a la función referencial (Bauman \& Briggs, 1990; Labov, 1972; Shiro, 1997). La función expresiva o evaluativa ha sido ampliamente estudiada, sobre todo en las últimas dos décadas. Estas investigaciones examinan la expresión de la afectividad en el discurso de hablantes monolingües en varios géneros discursivos (en inglés, Hunston \& Thompson, 2001; Sarangi, 2003; Martin \& White, 2005; Bednarek, 2008. En español, Bolívar, 2001 2006; Shiro, 2003; Kaplan, 2009; entre otros). Asimismo, se han hecho estudios comparativos entre varias culturas, para describir las diferencias de los usos entre hablantes de varias lenguas (Wierzbicka, 1999; Pavlenko, 2005). Sin embargo, 
se ha dicho muy poco acerca de la relación entre afectividad y producción lingüística en hablantes bilingües (Pavlenko, 2006; Koven, 2006) y menos todavía sobre cómo los niños desarrollan estas habilidades cuando están inmersos en dos grupos culturales.

Para poder comparar las expresiones de afectividad en dos lenguas, es necesario identificar las expresiones verbales que se van a comparar. En un enfoque integrador se podrían analizar las marcas de subjetividad en los enunciados (Hunston \& Thomson, 2001; Sarangi, 2003; Martin \& White, 2005) y se podría combinar el análisis de las formas lingüísticas con el nivel suprasegmental (entonación, calidad de la voz, etc.) y proxémico (gestos, postura, etc.). Este trabajo, sin embargo, se limitará a examinar las referencias explícitas a la afectividad, entendida ésta como la referencia a las emociones, a la volición y a la actitud epistémica en los enunciados que la madre dirige al niño.

Las preguntas que han guiado este trabajo son:

- ¿Qué expresiones de afecto usan las madres bilingües cuando se dirigen a sus hijos en cada una de las lenguas que dominan?

- ¿Cómo varía la frecuencia de estas expresiones según la lengua usada?

\section{Las expresiones de afectividad}

Para los fines de este trabajo, la afectividad se define como la referencia explícita a emociones, volición y actitud epistémica; basándose, entre otros, en la definición de Caffi y Jenney (1994) que conceptualizan la comunicación emotiva como la estrategia intencional de señalar información afectiva en la lengua oral y escrita (disposiciones evaluativas, compromisos evidenciales, actitudes de volición, orientaciones de relación, grados de énfasis, etc.).
Caffi y Jenney (1994) sostienen también que los hablantes de una lengua poseen algo que se puede llamar capacidad emotiva, refiriéndose a habilidades comunicativas básicas, convencionales $\mathrm{y}$ aprendidas, de tipo relacional y afectivo, que les permiten interactuar de manera fluida, negociar posibles conflictos interpersonales y lograr metas diferentes en el habla.

Si la capacidad emotiva es una habilidad que, como bien dicen Caffi y Jenny (1994), es aprendida y convencional, es decir, construida discursivamente (Ochs \& Schiefflin, 1989), cabe plantear la interrogante de cómo se desarrolla esta capacidad en cada hablante. En trabajos anteriores (Shiro, 2003; 2007) se ha mostrado que los niños monolingües hispanohablantes de primero y cuarto grado están en una etapa en la que estas habilidades todavía están en proceso de desarrollo y que los procesos evolutivos varían según el tipo de interacción o género discursivo que producen los niños. Pero, ¿qué pasa con los niños que están expuestos desde temprana edad a dos lenguas y, por tanto, a dos convenciones diferentes? ¿Cuál práctica discursiva prevalece?

Estudios recientes se plantearon esta interrogante en relación con los adultos bilingües. Pavlenko (2006) hace referencia a la "esquizofrenia" del bilingüe, puesto que algunos hablantes reportan que cambian de personalidad cuando cambian de idioma; otros dicen sentirse más cómodos hablando acerca de un tema en una de las lenguas que dominan y no tanto en la(s) otra(s). La experiencia emocional se refleja de manera diferente en lenguas diferentes: algunas personas se sienten emocionalmente estimuladas cuando se comunican en la lengua materna, mientras que no reaccionan de la misma manera en la segunda lengua (Harris et al., 2006). Muchos trabajos recogen la falta de equivalencia entre las 
expresiones que hacen referencia a las emociones y las dificultades de traducirlas de una lengua a la otra (Wierzbicka, 1999). Si las lenguas pueden moldear el pensamiento (Boroditsky, 2001; Evans \& Levinson, 2009; Lucy \& Gaskins, 2001), es de suponer que pueden moldear, de manera similar, la expresión de la afectividad (Panayiotou, 2006). Según esta autora (Panayiotou, 2006), algunas emociones que sobresalen en un grupo cultural, pueden incluso no existir en otro, lo cual evidencia que la afectividad se construye discursivamente. Dewaele y Pavlenko (2002) reportan diferencias en el vocabulario de las emociones de los hablantes de lengua materna y lengua extranjera. Es sabido, por ejemplo, que la emoción referida por "saudade" en portugués no transmite lo mismo que "nostalgia" en español, o que el estado emocional reflejado en la palabra "concern" en inglés, no es exactamente igual a "interés" o "preocupación" en español. Altarriba (2003) afirma que la palabra "cariño“, en español, no tiene un equivalente exacto en inglés, puesto que puede referirse a "liking" o a "affection". Asimismo, los diminutivos ("hijita") y las formas de tratamiento como "mi amor", que se usan para dirigirse a alguien cariñosamente, difieren de un grupo social y cultural a otro, hasta en la misma lengua: en Venezuela, apelativos de tratamiento cariñoso entre los interlocutores incluyen términos como "negro", "negrito" o "gordo", "gordito", que se consideran ofensivos en otras regiones hispanohablantes.

De todo esto se desprende que la expresión de las emociones depende de las normas sociales de uso de la lengua, puesto que se construyen y reconstruyen en ella porque la experiencia corporal cruda de una emoción debe ser filtrada a través de un sistema cultural de significados, es decir, el lenguaje (Panayiotou, 2006).
De esta manera, el hablante bilingüe no es equivalente a dos monolingües en un solo cuerpo y tampoco se puede equiparar con un único monolingüe, en el que se combinan dos lenguas para formar un todo (Pavlenko, 2006).

Cabe preguntarse entonces, ¿qué ocurre con los niños que crecen en entornos donde están expuestos a más de una lengua? Sabemos que hoy en día, resultado de la globalización y de las migraciones, es cada vez menos común que los niños crezcan en un ambiente monolingüe. Tomemos el caso particular del sur del estado de la Florida en Estados Unidos. Por su cercanía con Latinoamérica, por las olas migratorias y porque en un tiempo fue colonia española, un alto porcentaje de la población habla inglés y español. Según las estadísticas (Pew Research Hispanic Center, 2013), la constitución demográfica es tal que casi $20 \%$ de la población de Florida nació fuera de los Estados Unidos y la gran mayoría proviene de países de América Latina (la concentración de hablantes bilingües español-inglés debe ser considerablemente mayor en el sur del estado, en la ciudad de Miami y sus alrededores, puesto que ahí residen o visitan muchos latinoamericanos). En este sentido, esta región ofrece un contexto ideal para investigar el desarrollo de lenguaje en entornos bilingües.

\section{Método}

Para responder a las preguntas que guiaron esta investigación, se recopiló un corpus de interacciones espontáneas entre 10 madres bilingües, residentes del sur de la Florida (español la lengua materna e inglés la segunda lengua, en adelante L2) y 10 madres angloparlantes monolingües (en adelante, inglés L1) con sus hijos de 30 meses. Se seleccionaron las interacciones de madres con hijos de 30 meses puesto que había que mantener la edad de los 
niños constante para eliminar la variación del habla de la madre en función de la edad (y, por tanto, de las destrezas comunicativas) del niño que participa en la interacción (finetuning, Snow 1995). La evidencia que las madres (y los hablantes, en general) adaptan su producción lingüística a las necesidades de sus hijos hace suponer que la afectividad en el habla de la madre varía también en función de la edad de su hijo o hija.

Asi mismo, se buscaba analizar las interacciones a una edad temprana (y 30 meses era la edad más temprana a la que se tenía acceso a interacciones equivalentes con participantes similares), para poder seguir en futuras investigaciones, de manera longitudinal, cómo evoluciona el uso de estas expresiones en el lenguaje infantil así como en el habla de la madre.

Tanto en el grupo bilingüe como en el monolingüe, las madres provenían de un estrato social medio-alto y habían completado sus estudios de secundaria. La mayoría cursó varios años de estudios universitarios. Las madres hispanohablantes nacieron en algún país de América Latina (Colombia, República Dominicana, Perú y Puerto Rico) y sus edades oscilan entre 28 y 38 años. Salvo una de ellas que llegó a Estados Unidos desde Puerto Rico a los 3 años y medio ${ }^{3}$, todas llegaron adultas

\footnotetext{
${ }^{3}$ La decisión de incluir a esta participante se tomó según dos criterios, uno práctico y otro teórico: por una parte, los valores de las expresiones de afectividad de sus transcripciones eran bastante similares al promedio del grupo de madres bilingües y, por otra, según Pedraza (1985) y Urciuoli (1996), los puertorriqueños que se establecen en el Sur de la Florida tienden a mantener el español como lengua de herencia más que otros grupos provenientes de regiones diversas de América Latina. En el contexto sociolingüístico del Sur de la Florida, el uso del español es tan extendido (Montrul, 2013) que es difícil medir el grado de bilingüismo de un hablante por los años de residencia en esta región.
}

(y llevan aproximadamente una década en el país). Las madres angloparlantes, cuyas edades oscilan entre 27 y 40 años, participaron en una interacción espontánea en la que conversaban con su hijo o hija mientras jugaban con 3 bolsas de juguetes: una que contenía figuras de animales, otra que tenía juguetes que representaban comida y una tercera bolsa que contenía libros para leer. Las madres bilingües participaron en dos conversaciones cada una, una en español y otra en inglés (L2), usando las mismas tres bolsas de juguetes. Las 30 interacciones fueron grabadas en video y audio, transcritas en formato CHAT (Codes for the Human Analysis of Transcripts, Sokolov y Snow 1994) y codificadas para su posterior análisis con la ayuda del programa CLAN (Child Language Analysis, MacWhinney, 2000). Las madres recibían instrucciones para interactuar con sus hijos en la lengua indicada (español o inglés L1 o L2) como lo hacían habitualmente. Cabe mencionar que todas las madres bilingües seleccionadas para este estudio manifestaron que acostumbraban conversar tanto en español como en inglés con sus hijos, de modo que las interacciones analizadas se asemejan a la manera habitual de comunicarse en estos hogares.

Con el propósito de responder a las preguntas de investigación, se identificaron las expresiones de afectividad que se definieron como pertenecientes a las funciones expresivas del lenguaje y que tienen un papel evaluativo en la interacción. Siguiendo los trabajos de Shiro (1997, 2003 y 2007), se agruparon las expresiones de afectividad en tres grandes dimensiones: la referencia explícita a las emociones, a la actitud epistémica y a la volición. La referencia explícita ${ }^{4}$

\footnotetext{
${ }^{4}$ Ver Oatley y Jenkins (1996) y Bednarek (2008) para una revisión detallada de las emociones.
} 
a las emociones implica que se nombra algún sentimiento, estado de ánimo o emoción, por ejemplo, "Mami se pone brava si pones el juguete en la boca". Se agruparon las expresiones referentes a las emociones en las siguientes subcategorías: alegría, placer, cariño, miedo y sorpresa. Para cada una se indicaba si se trataba de una expresión positiva o negativa. Así “¿por qué estás triste?” se codificó como alegría negativa y "Te quiero mucho" como cariño positivo. La volición explícita requiere la presencia de un verbo que indique voluntad o deseo: "¿Te gustaría jugar conmigo?" 5 . Por último, la actitud epistémica que se tomó en cuenta se expresa mediante la presencia de un verbo que refiere a un proceso mental: "Creo que el pato nada en el agua", de un adverbio que señala grado de certeza ("Probablemente se quedó dormido") o de un verbo modal ("No pise la tableta que se puede romper") ${ }^{6}$. Adicionalmente, para cada uno de los códigos de afectividad se especificó si la perspectiva que se adoptaba era del hablante, es decir la madre; del oyente, es decir, del niño o la niña que participaba en la interacción; de ambos, es decir, de "nosotros" inclusivo (madre e hijo o hija); o de alguien o algo mencionado en el enunciado. De esta manera, es posible determinar si la madre usa con mayor frecuencia expresiones que hacen referencia a la afectividad

\footnotetext{
${ }^{5}$ Nótese que esta pregunta hace referencia a la volición, mientras que "¿Te gusta jugar conmigo?" se refiere a lo que denominamos emoción.

${ }^{6}$ Según Shiro (2007) la actitud epistémica se expresa por otros medios también, no solo con la presencia de los verbos que indican procesos mentales, pero la presencia de estos verbos indica que el hablante no tiene certeza absoluta del contenido de su enunciado (Por ej., "Recuerdo que el día de mi cumpleaños llovió" señala menos certeza que "El día de mi cumpleaños llovió). Para un análisis más exhaustivo del desarrollo de las expresiones de actitud epistémica, ver Shiro (2007).
}

propia, a la de su hijo, hija o a la de otros.

Finalmente, se tomaron en cuenta algunas formas lingüísticas que indican claramente afectividad, pero que se pudieron incluir en las categorías arriba mencionadas. Ese es el caso de los diminutivos ("el patito"), formas lexicales usadas típicamente con bebés ("dame el tete"), expresiones de cortesía ("por favor", "gracias") y formas de tratamiento: por el nombre "ven, José", por un apodo ("ven, Pepito") o un término cariñoso ("ven, mi amor"). La decisión de incluir estas formas se basa en la comparación de estos mismos enunciados cuando se omiten las formas identificadas y se observa que el estilo se vuelve menos afectuoso, va más directo a los hechos. Las expresiones de cortesía, los diminutivos y los términos de tratamiento cumplen una función evaluativa (no referencial) que promueve la interacción a nivel interpersonal (Verschueren, 1999), que es justamente el foco de esta investigación.

Análisis e interpretación de los datos

Para responder a las preguntas planteadas, en primer lugar se comparó el número total de expresiones de afectividad usadas por las madres nativas del español cuando se dirigen a sus hijos en español y en inglés (L2) y de las madres nativas del inglés (L1). Como se observa en la figura 1, las madres nativas del inglés (L1) usan un número considerablemente menor de expresiones de afectividad. Aun cuando esta diferencia no es estadísticamente significativa, interesa examinar la producción verbal de las madres como muestra de la experiencia a la cual están expuestos los niños en un contexto bilingüe. Se observa que la cantidad de expresiones de afectividad usada por las madres nativas del español es mayor, tanto en español como en inglés (L2), que la cantidad de las expresiones 


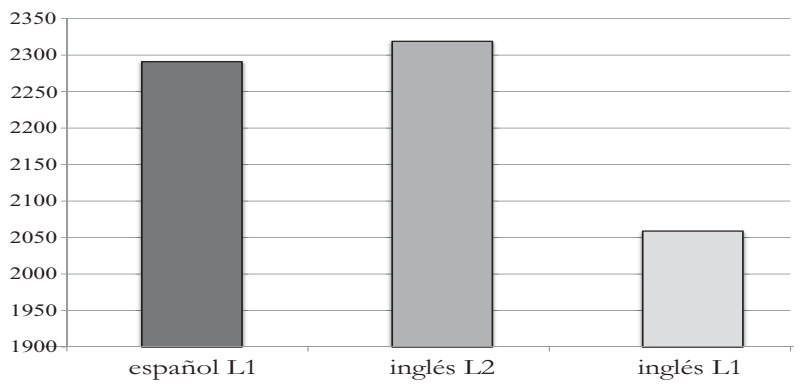

Figura 1. Total expresiones de afectividad en los tres grupos

usadas por las madres angloparlantes, razón por la cual se supone que los hijos de madres bilingües oyen un número mayor de expresiones de afectividad, en interacciones similares, que los hijos de madres angloparlantes.

Sin embargo, es necesario tomar en cuenta que la extensión de las interacciones varía considerablemente entre los grupos: las madres nativas del español producen un promedio de 894 enunciados (mínimo 716, máximo 1159), en español, y 765 (mínimo 459, máximo 948) en inglés (L2), mientras que las madres angloparlantes producen un promedio de 797 enunciados (mínimo 515, máximo 1033). Si se quiere controlar el largo de la interacción de las madres, para confirmar que la diferencia no se debe a que las interacciones en inglés (tanto nativo como no nativo) tienden a ser más cortas

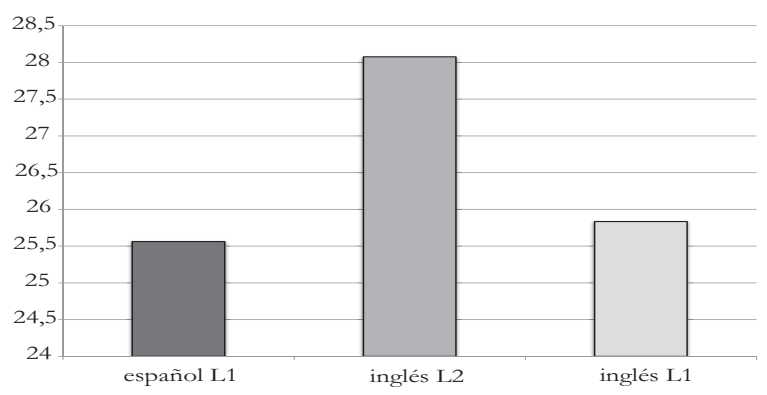

Figura 2. Porcentaje de expresiones de afectividad del total de enunciados. que las interacciones en español, es necesario calcular el porcentaje de enunciados codificados del total de enunciados en cada interacción. La figura 2 muestra el resultado.

Ciertamente el panorama en la figura 2 cambia, puesto que la proporción de expresiones de afectividad en las interacciones en la lengua nativa, tanto en español como en inglés (L1), es bastante similar. Sin embargo, el porcentaje de expresiones de afectividad en las interacciones en inglés (L2) de las madres hispanohablantes supera con creces al de las madres nativas. Los datos del gráfico 2 implican que, en líneas generales, si se considera que la frecuencia de las expresiones de afectividad es reflejo de las normas culturales, no hay grandes diferencias entre los dos grupos de hablantes nativos, puesto que el porcentaje de expresiones de afectividad, que no sobrepasa un tercio de los enunciados, es similar en las interacciones en la lengua materna tanto de las nativas del inglés como en las de las nativas del español, y es un poco mayor en la conversación en inglés (L2) de las hispanohablantes.

¿Qué ocurre si se desglosa con mayor detalle el uso de esas expresiones? Se ha visto que las expresiones de afectividad fueron agrupadas en tres dimensiones: la referencia explícita a las emociones ("emoción"), a la voluntad o el deseo ("volición") y a la creencia o al conocimiento ("epistémico").

En la figura 3 se observa que todas las madres usan un número considerablemente mayor de expresiones de emoción que de expresiones referidas a las actitudes epistémicas y a la volición. Asimismo, se detectan diferencias notables entre los dos grupos (nativas del español y del inglés), sobre todo en el uso de las expresiones de emoción. Se puede notar que las madres hispanohablantes, cuando hablan 


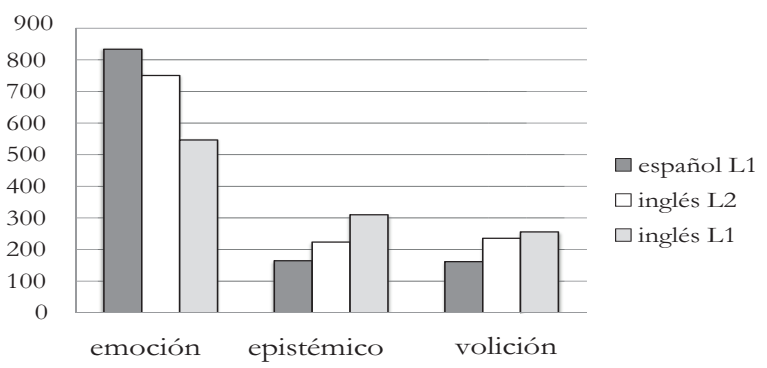

Figura 3. Número de expresiones de emoción, actitud epistémica y volición

en su lengua materna (español L1), hacen referencia a las emociones $37 \%$ más que las madres angloparlantes (inglés L1) y, cuando hablan en inglés (L2), usan 50\% más expresiones de emoción que sus pares angloparlantes, aproximándose más a su actuación en español y no tanto a la norma cultural reflejada en las interacciones de las madres nativas del inglés. En cambio, las madres nativas del inglés usan un mayor número de expresiones de actitud epistémica y de volición que las madres nativas del español, cuando estas últimas interactúan en español. El número de expresiones de actitud epistémica y de volición en las interacciones en inglés (L2) de las nativas del español, aun cuando es un poco menor que el de sus pares nativas del inglés (L1), se acerca más en cantidad a esas últimas y es considerablemente mayor que el número de expresiones usadas en español. Esto parece indicar que las madres nativas del español, usan las expresiones de actitud epistémica y volición de manera similar al uso de las madres angloparlantes.

\section{La figura 4 muestra el porcentaje delos tres tipos}

\footnotetext{
${ }^{7}$ Las diferencias entre los datos arrojados por los gráficos $1 \mathrm{y}$ 3 , por el número de casos, y los arrojados por los gráficos 2 y 4 , por los porcentajes, no son grandes. Sin embargo, es necesario tomar en cuenta ambos resultados, puesto que los porcentajes
}

de afectividad ${ }^{7}$ que se identificaron en el habla dirigida a los niños de madres bilingües, españolinglés, y madres monolingües angloparlantes, ya que así se logra controlar nuevamente el efecto de la extensión de las interacciones. En el gráfico 4 se observa que las tendencias permanecen similares a lo reflejado en el gráfico anterior: las madres angloparlantes hacen un uso menos frecuente de las expresiones de emoción, que las hablantes nativas en español cuando hablan en inglés (L2) o en español, pero usan con mayor frecuencia las expresiones epistémicas y de volición que las nativas del español cuando hablan en su lengua materna. En lo que sigue me planteo analizar cada tipo de afectividad para poder explicar con mayor detalle las diferencias entre los tres grupos de interacciones.

\section{Expresiones de emoción}

Para determinar las normas culturales con respecto a las expresiones de emoción, es posible comparar las hablantes nativas, del inglés

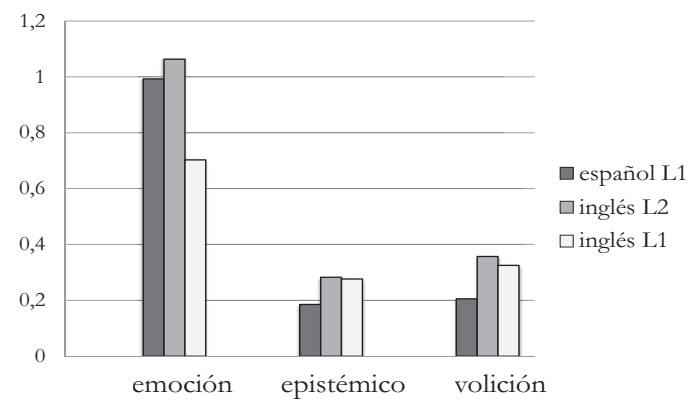

Figura 4. Porcentaje de expresiones de emoción, actitud epistémica y volición del total de los enunciados

informan acerca de las tendencias del grupo cultural (monolingüe o bilingüe, español o inglés), los números de casos dan una idea de la muestra de habla a la cual están expuestos los niños. Si bien el objetivo de este estudio se refiere a lo primero, investigaciones futuras sobre el desarrollo de estos aspectos en la producción de lenguaje de los niños tendrán que tomar en cuenta la experiencia a la que están expuestos los niños, y el número de casos, más que el porcentaje, forma parte de esa experiencia. 
y del español. Por una parte, se observa que hay una gran diferencia numérica entre las madres hispanohablantes (834 enunciados que contienen referencia a las emociones) y las angloparlantes (546 enunciados con referencia a las emociones). Por la otra, los porcentajes de las expresiones de emoción de las hablantes nativas del español $(9 \%)$ y de inglés $(7 \%)$ son bastante similares (la diferencia no es estadísticamente significativa), lo cual podría indicar que las normas culturales que guían las prácticas discursivas relacionadas con la referencia a las emociones en estos dos grupos son bastante similares. Sin embargo, cuando se comparan las expresiones referentes a las emociones de las madres hispanohablantes en inglés (L2) con las de las madres angloparlantes, se encuentra que las hablantes de inglés L2 usan significativamente más referencias a las emociones que las madres hablantes de inglés L1 $\left(F_{1,18}=5.40, p<.032\right)$.

Un análisis cualitativo nos muestra, igualmente, un panorama con diferencias fundamentales, sobre todo en el plano discursivo y lexical. Desde el punto de vista discursivo, las referencias a las emociones en las interacciones de las hablantes nativas del español (tanto en español como en inglés) se pueden convertir en el tema de la conversación, como se refleja en los siguientes ejemplos:

Ejemplo $1^{8}$

(madre nativa del español conversa en español)

*MOT: ése es un pato.

${ }^{*}$ CHI: pato.

*MOT: si.

*MOT: dale, dale un besito al gallito.

\footnotetext{
${ }^{8}$ La transcripción se hizo en formato CHAT (MacWhinney 2000), pero se eliminaron los símbolos que no eran pertinentes para este análisis.
}

*MOT: pobrecito.

*MOT: está solito.

*MOT: dale un besito.

*MOT: dale un besito.

*MOT: no?

*MOT: no le quieres dar un besito?

${ }^{*}$ CHI: yeah.

*MOT: si?

*MOT: está como llorando.

*MOT: muestra.

*MOT: mira.

*MOT: mírale los ojos.

*MOT: está llorando?

*CHI: yeah.

*MOT: dale un besito.

Ejemplo 2

(madre nativa del español conversa en inglés L2)

*MOT: pero mama wants, mommy want a kiss. [pero mamá quiere, mami quiere un beso]

*MOT: I want a hug. [quiero un abrazo]

*CHI: mi fish! [jmi pez!]

*MOT: I'll give it to you. [te lo daré]

*MOT: but if you give me a kiss. [pero si me das un beso]

*MOT: give me a kiss. [dame un beso]

*CHI: mi fish? [¿mi pez?]

*MOT: your fish? [¿tu pez?]

*CHI: sí. [si]

*MOT: is here. [está aquí]

\%com: grabsthefish. [agarra el pez]

*MOT: is here. [está aquí]

*MOT: no! [jno!]

*MOT: give me a kiss. [dame un beso]

*CHI: 0 [=! gives kiss]. [le da un beso]

*MOT: give me a hug. [dame un abrazo]

*CHI: no! [jno!

*MOT: ay. [ay]

*MOT: yes! [jsí!] 
*CHI: dame! [jdame!]

*MOT: give me a hug! [dame un abrazo]

${ }^{*}$ CHI: 0 [=!giveshug]. [le da un abrazo]

*MOT: love you! [jte quiero!]

*MOT: I loveyou. [yo te quiero]

*MOT: you love me? [¿tú me quieres?]

*CHI: hm! [jhm!]

*MOT: you love me? [¿me quieres?]

\%com: motherkisseschild. [la madres besa al niño]

*MOT: you love me? [¿me quieres?]

*MOT: toma. [jtoma!]

*MOT: say thank_you mom. [di "gracias, mami”]

*CHI: thank_you mom. [gracias mami]

*MOT: xxx.

\%com: more than two unintelligible words spoken. [se produjeron por lo menos dos palabras ininteligibles]

*MOT: you love mama? [¿tú quieres a mamá?]

*CHI: sí [sí]

*MOT: how much? [¿cuánto?]

*CHI: xx much. [mucho]

*MOT: how much you love me? [¿cuánto me quieres tú a mí?]

*CHI: xxx.

*MOT: tell me. [dime]

*MOT: I need to know. [necesito saber]

${ }^{*} \mathrm{CHI}$ : xxx.

*MOT: tell me. [dime]

*MOT: I need to know. [necesito saber]

*MOT: how much you love me, how much you love me? [¿cuánto me quieres, cuánto me quieres?]

*MOT: di a lot. [di, un montón!]

*CHI: hm. [hm]

*MOT: di a lot. [di, un montón!]

*CHI: hm. [hm]

En el ejemplo 1, la secuencia del beso y del llanto es temática, y se encuentran secuencias similares en otras transcripciones en español así como en inglés (L2), lo cual indica que puede ser una rutina recurrente en ciertas circunstancias de las madres nativas del español. El ejemplo 2, en cambio, es un caso especial en el que la madre hispanohablante le dedica una buena parte de la conversación en inglés (L2) al tema del cariño que la une a su hijo.

En ninguna de las interacciones de las madres angloparlantes se encuentran secuencias temáticas similares, dedicadas a la emoción. Las marcas de emoción se esparcen a lo largo de la transcripción y hacen más referencia a la satisfacción, o la falta de ella ${ }^{9}$, que a expresiones de cariño o amor, como se observa en el siguiente ejemplo:

Ejemplo 3

(madre nativa del inglés L1)

*MOT: [- eng] here's one of your favorites. [aquí está uno de tus favoritos]

*MOT: [- una] oh. [oh]

*MOT: [- eng] you closed it? [¿lo cerraste?]

*MOT: [- eng] good job. [iqué bien!]

*CHI: [- una] yay. . [iyupi!]

*MOT: $+<$ [- eng] look. [mira]

*MOT: [- una] yay. [iyupi!]

*MOT: [- eng] you did it. [lo hiciste]

*CHI: [- eng] it's open! [está abierto]

*MOT: $+<$ [- eng] look at this. [mira esto]

*MOT: [- eng] yup. [sí]

*MOT: [- eng] it's open. [está abierto]

*CHI: [- eng] I close. [lo cierro]

\footnotetext{
${ }^{9}$ En la codificación, estos enunciados que expresan satisfacción (o insatisfacción) se identificaron como "placer" (positivo o negativo) dentro de la dimensión de la expresión de las emociones, a diferencia de "afecto" que se refiere a expresiones de amor y de cariño.
} 
*MOT: [- eng] do you like apples? [¿te gustan las manzanas?]

*CHI: [- una] yummy. [mmmm]

*MOT: [- eng] don't eat it, honey. [no te las comas, cariño]

*MOT: [- eng] it's just pretend. [son de mentira]

En el ejemplo 3, la madre nativa del inglés (L1) hace énfasis en lo que le gusta al niño o a ella misma. La única marca de cariño es el apelativo "honey" que en nuestro análisis se incluyó en una categoría aparte a la cual se hará referencia en lo que sigue.

La segunda diferencia cualitativa en la referencia a las emociones es el uso del léxico. Se comparó el uso de ciertas palabras de cariño (amor, beso, abrazo, love, kiss, hug, sweetie, honey) en los tres grupos y se encontró que las hablantes nativas del inglés no usan "kiss" y "hug" en ninguna instancia, mientras que todas las participantes nativas del español usaron estos dos términos de afecto (por lo menos una vez), o su equivalente en español ("beso" y "abrazo"), tanto en las interacciones en su lengua materna como en inglés (L2).

La figura 5 muestra el número total de casos (tokens) de las palabras de cariño incluidas en la lista arriba mencionada y evidencia la gran diferencia en los usos de estos elementos lexicales. Las madres nativas del español usan siete veces más estos vocablos, tanto en español como en

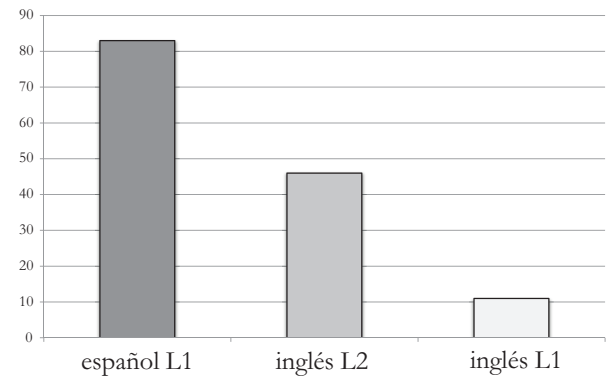

Figura 5. Léxico de la emoción inglés, que las madres nativas del inglés. Llama la atención que los pocos casos utilizados por las madres angloparlantes se reducen a varias formas del lexema "love" que, como se sabe, no equivale a "amor" en todos los contextos puesto que, usado como verbo, "love" puede expresar placer o satisfacción (por ejemplo, "I love apples" cuyo equivalente en español sería "Me encantan las manzanas" o "Me gustan mucho las manzanas" y no necesariamente "Yo amo las manzanas").

En resumen, la conclusión que se puede derivar de este análisis es que las convenciones relacionadas con las expresiones de emoción difieren entre los grupos angloparlantes e hispanohablantes y, cuando una madre nativa del español se dirige a su hijo o hija de 30 meses en inglés, sus expresiones emotivas se asemejan al uso de su lengua materna, el español, más que a las prácticas discursivas del inglés.

\section{Expresiones de actitud epistémica}

En el caso de las expresiones de actitud epistémica, los resultados muestran que, pese al uso poco frecuente en las tres muestras (español L1, inglés L1 e inglés L2), existe una diferencia entre las madres angloparlantes e hispanohablantes cuando interactúan en su lengua materna. Las madres nativas del inglés usan un porcentaje significativamente mayor de expresiones de actitud epistémica $\left(F_{1,18}=5.63, p\right.$ $<$.029) que las madres hispanohablantes.

De nuevo se encuentran diferencias en las normas culturales del español y del inglés. Se observa, sin embargo, que, a diferencia de las expresiones de emoción, el uso de las expresiones epistémicas en inglés de las nativas del español se asemeja al uso de las angloparlantes y se diferencia significativamente de la lengua 
materna, el español $(t(19)=-1.787, p<0.1)$, desde el punto de vista cuantitativo.

Se recurre al análisis cualitativo para determinar en qué consisten las diferencias entre el uso nativo y no nativo del inglés.

Ejemplo 4

(madre nativa del español conversa en inglés L2)

*MOT: [- eng] I'm looking for a dolphin. [estoy buscando un delfín]

*MOT: [- eng] where is the dolphin? [¿dónde está el delfín?]

*CHI: [- eng] okay. [ok]

*MOT: [- una] hm@fp.

*MOT: [- eng] I think it's in this area. [creo que por aquí]

*MOT: [- eng] you found the dolphin! [jencontraste el delfín!]

*MOT: [- eng] where is his house [=! points]? [¿dónde está la casa?]

*MOT: [- eng] in thegrass? [¿en la grama?]

*MOT: [- eng] or in the water? [¿en el agua?]

*CHI: $+<[-$ eng] that's not the dolphin. [esto no es un delfín]

*MOT: [- eng] it's not a dolphin? [¿no es un delfín?]

${ }^{*}$ CHI: [- eng] no. [no]

$*$ MOT: $+<$ [- eng] I think this is the dolphin. [yo creo que es un delfín]

*CHI: [- eng] no. [no]

*CHI: [- eng] that's [/] that's Shamu. [este, este es Shamu]

*MOT: [- eng] okay. [ok]

*MOT: [- eng] that's Shamu. [es Shamu]

*CHI: [- eng] that's the dolphin. [ése es el delfín]

*MOT: [- eng] I think that's a shark. [yo creo que es un tiburón]

Ejemplo 5

(madre nativa del inglés L1)

*MOT: [- eng] I can't understand what you're saying when you're eating buddy... [no puedo entender lo que estás diciendo mientras estás comiendo, amigo]

*MOT: [- eng] do you remember what it's called? [¿te acuerdas cómo se llama?]

*MOT: [- eng] what do you think that is?... [¿qué te parece que es esto?]

*MOT: [- eng] you let me know if you need some help. [déjame saber si necesitas ayuda]

En el extracto del ejemplo 4, la madre nativa del español usa tres expresiones que reflejan actitud epistémica y llama la atención que las tres tienen exactamente la misma forma. Revisando la transcripción en su totalidad, es posible darse cuenta que el verbo "think" y en menor grado "know" son los únicos que aparecen en la transcripción para expresar actitud epistémica. Las madres nativas del inglés, en cambio, varían los verbos que usan (think, know, remember, understand, etc.).

Es de suponer, entonces, que las madres hispanohablantes tienden a usar un número menor de verbos mentales que las madres angloparlantes cuando se dirigen a sus hijos de 30 meses en español y el uso de estos verbos aumenta considerablemente cuando se dirigen a sus hijos en inglés (L2), lo cual se aproxima a la actuación de las madres nativas del inglés (L1) desde el punto de vista cuantitativo. La diferencia cualitativa consiste en que las madres nativas del inglés (L1) hacen uso de un léxico más variado para expresar la actitud epistémica que las madres nativas del español cuando interactúan en inglés (L2).

\section{Expresiones de volición}

La diferencia en porcentaje de las expresiones de volición entre las nativas del inglés y español cuando usan su lengua materna es nuevamente significativa $\left(\mathrm{F}_{1,18}=8.53, p<.009\right)$, lo cual indica que las normas culturales que siguen las madres 
hispanohablantes no se parecen a las normas culturales de las madres angloparlantes, puesto que estas últimas hacen referencia explícita a la voluntad o al deseo con mayor frecuencia cuando se dirigen a sus hijos.

Cuandosecomparanlas expresiones devolición de las madres nativas del español cuando hablan en inglés (L2) con sus interacciones en español, la diferencia es muy grande y estadísticamente significativa $\left(t_{19}=-3.1, p<0.01\right)$, lo cual implica que en el uso de la volición, en las interacciones en inglés (L2), las madres hispanohablantes se asemejan más a las nativas del inglés (L1) que al uso de su lengua materna. Se recurre al análisis cualitativo para explorar si hay diferencias entre el inglés no nativo y nativo, y se encuentra que el uso del "nosotros inclusivo" es más frecuente en las madres nativas del inglés (L1), como se refleja en el siguiente extracto:

Ejemplo 6

(madre nativa del inglés L1)

*MOT: let's see what's in here. [veamos qué hay aquí adentro]

*MOT: ooh [/] ooh. [joh!]

*MOT: look at all this stuff. [mira todo esto]

*MOT: okay. [ok]

*MOT: what should we do with it? [¿qué deberíamos hacer con esto?]

*MOT: what do you wanna make? [¿qué quieres hacer?]

*CHI: a barn. [un establo]

*MOT: a barn? [¿un establo?]

*MOT: okay. [ok]

*CHI: yeah. [sí]

*MOT: go ahead. [dale]

*MOT: let's see.[veamos]

Ejemplo 7

(madre nativa del español conversa en inglés)

*MOT: but I want you to put those inside... [pero yo quiero poner esos adentro]
*MOT: I want you to put the palm and close to this one $[=!$ points]... [quiero que tú pongas la palmera y que cierres esto]

*MOT: I want them in the corner... [los quiero en la esquina]

En el ejemplo 7 se nota el uso de la expresión "I want" o "I want you to...", expresión que usaron las 10 madres hispanohablantes cuando conversaban en inglés (L2). De las madres angloparlantes, una sola usó dicha expresión en dos ocasiones. Es posible que este uso, que no es frecuente en inglés porque se considera demasiado impositivo sea una traducción literal del español de "yo quiero que (tú)...", pero usado en inglés (L2) cae fuera de la norma cultural establecida y se interpreta de una manera distinta tal vez a la intención de la hablante.

Otras formas usadas para expresar afectividad

Por último, en el gráfico 6 se observa el análisis de algunas formas que expresan afectividad, el sufijo diminutivo y los apelativos, y que no se han incluido en las tres categorías arriba analizadas (emoción, actitud epistémica y volición).

Como es de esperarse, sobresale el uso del diminutivo como el de mayor frecuencia en español, pero con muy poca frecuencia en inglés (L1 y L2). Este contraste es meramente lingüístico, puesto que se debe a las características inherentes de las dos lenguas: a diferencia del español (Lázaro Mora, 1999), en inglés el sufijo diminutivo es poco frecuente y su uso es muy marcado, limitado al lenguaje usados con bebés (babytalk, Quirk et al., 1989). La figura 6 indica que las madres nativas del inglés (L1) recurren poco al uso del diminutivo cuando se dirigen a sus hijos de 30 meses. Las madres nativas del español usan el diminutivo en inglés (L2) dos veces más que las nativas del inglés, lo cual sugiere que retienen hasta cierto puntlos rasgos 
de su lengua materna, una interferencia nada sorprendente en usuarios de una segunda lengua.

La otra diferencia marcada, esta vez puramente pragmática, puesto que no se relaciona con las características formales del español o del inglés, es el uso de los apelativos, es decir usar la forma vocativa para llamar al niño o niña por su nombre (por ejemplo, "siéntate aquí, Mateo", “¿qué es esto, Adrianita?”), o con una palabra afectuosa (“¿qué quieres comer, papito?”). Las madres nativas del español usan el apelativo tres veces más en español (una diferencia estadísticamente significativa $\left(F_{1,18}=7.16, p<\right.$ .015), y cuatro veces más en inglés (L2), que las madres angloparlantes, lo cual implica que es una práctica discursiva común de las hablantes nativas del español tanto cuando se comunican en la lengua materna como cuando lo hacen en la segunda lengua.

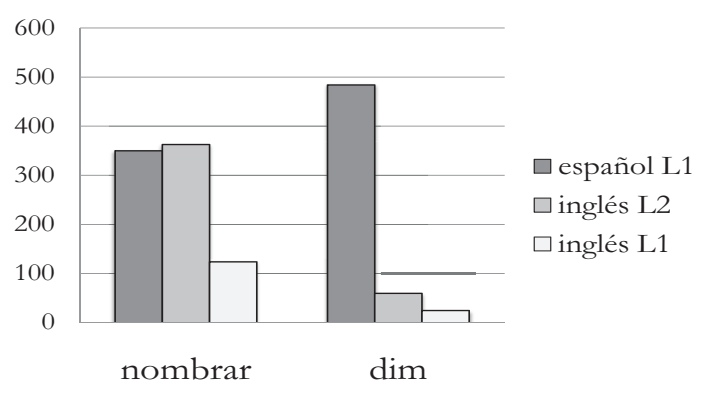

Figura 6. Formas lingüísticas que expresan afectividad

\section{Conclusiones}

De este análisis se derivan varias conclusiones de tipo teórico y metodológico. En primer lugar, se evidencia que los niños que crecen en entornos bilingües están expuestos a normas discursivas híbridas que no se limitan a las interferencias formales tan bien documentadas de los hablantes no nativos como son las interferencias en el nivel fonológico, morfosintáctico o léxico. Nuestros resultados sugieren que las diferencias culturales dejan huella en el uso de las expresiones de afectividad, huellas que varían según el tipo de afectividad que se considere. En las expresiones de actitud epistémica y de volición, los contrastes entre nativos y no nativos en inglés no son tan marcados, pese a que el uso nativo del español se diferencia claramente del uso nativo del inglés. En el ámbito de las emociones, en cambio, el uso no nativo del inglés se diferencia marcadamente del uso nativo (sobre todo en el aspecto cualitativo), asemejándose más a las normas culturales del español nativo de las participantes. Estos resultados indican que solo una pequeña parte de la variación en la expresión de la afectividad se debe a rasgos formales que distinguen las lenguas comparadas, como por ejemplo el caso del diminutivo del español. Se ha mostrado aquí que el "acento extranjero" asume formas distintas, a veces pragmáticas, a veces discursivas, pocas veces analizadas en la bibliografía especializada, y no se limitan a la pronunciación, al vocabulario o a la sintaxis, aspectos que han sido muy documentados en el campo del bilingüismo (Hoff et al., 2012; Hoff, 2013).

Es oportuno mencionar que los datos de este estudio (10 participantes bilingües y 10 monolingües) no son suficientes para suponer que los resultados aplican a toda la población bilingüe español-inglés, ni siquiera a la población bilingüe del sur de la Florida, dada la gran diversidad de país de origen, nivel de educación, estrato socioeconómico, tiempo de estadía en los Estados Unidos, todos estos factores que influyen en los aspectos aquí estudiados. No obstante, la muestra analizada es bastante homogénea como para reflejar ciertas tendencias que se 
deben comprobar en futuras investigaciones con corpus de mayores proporciones.

En segundo lugar, se evidencia que para estudiar ciertos fenómenos del lenguaje es necesario recurrir tanto a métodos cuantitativos como cualitativos. El método cuantitativo permite detectar las tendencias basadas en la frecuencia de los casos, pero pierde de vista ciertos aspectos que la frecuencia sola no explica y que resaltan únicamente cuando se hace un análisis exhaustivo de ciertas combinaciones discursivas o de la presencia o ausencia de algún elemento en particular. El análisis cualitativo se hace más necesario todavía en estudios sobre bilingüismo, puesto que permite tomar en cuenta el contexto necesario para asegurarse de que los elementos cotejados son equiparables desde el punto de vista funcional $y$, por tanto, pueden ser considerados equivalencias de traducción.

En este estudio se ha adoptado una perspectiva centrada en las marcas lingüísticas de las expresiones de la afectividad. Se han tomado en cuenta las características demográficas de las madres participantes en cuanto a nivel de educación, estrato socioeconómico, lengua materna y edad del niño con quien interactuaban. Corresponde a futuras investigaciones incluir otros factores que posiblemente inciden en el uso de las expresiones de la afectividad. Basados en estudios anteriores sobre monolingües (Shiro, 2003), es posible suponer que el tipo de interacción incide en el uso del lenguaje expresivo $^{10}$. Asimismo, factores como el sexo del niño a quien se dirige la madre, el nivel de competencia que alcanza la madre en la lengua no nativa, entre otros, podrían tomarse en cuenta para explicar la variación discursiva (Dewaele \& Pavlenko, 2002).
Analizar el habla que las madres bilingües dirigen a sus hijos de 30 meses permite vislumbrar una componente importante del discurso al cual están expuestos los niños, especialmente los de temprana edad, que crecen en entornos donde se manejan dos lenguas porque se basa en el supuesto de que los niños pasan la mayor parte del tiempo al cuidado de la madre. Adicionalmente, habría que determinar hasta qué punto influyen los otros hablantes, bilingües o no, en los procesos evolutivos del discurso infantil. Indudablemente, para comprobar si las expresiones de la afectividad en el discurso infantil siguen normas culturales híbridas similares a las encontradas en el discurso materno, es indispensable enfocarse en la producción discursiva de los niños inmersos en un contexto bilingüe, objetivo que se propone plantear en la segunda etapa de esta investigación.

\section{Referencias}

Altarriba, J. (2003). Does “cariño" equal "liking"? A theoretical approach to conceptual nonequivalence between languages. International Journal of Bilingualism, 7, 305-322.

Bauman, R. \& Briggs, C. (1990). Poetics and performance as critical perspective on social life and language. Annual Review of Anthropology, 19, 59-88.

Bednarek, M. (2008). Emotion talk across corpora. London: Palgrave.

Bolivar, A. (2001). The negotiation of evaluation in written text. En M. Scott y G. Thompson (eds.), Patterns of text: In honour of Michael Hoey, (pp. 129158). Amsterdam: John Benjamins.

Bolívar, A. (2006). La función de la evaluación en artículos y ensayos humanísticos. En J. Falk y

${ }^{10}$ Compárese "No metas el dedo en la boca, mi amor" (con intención de disciplinar) con "¿Quieres un helado, mi amor?" (con intención de complacer). 
F. Weichmeister Bermúdez (coords.) Discurso, interacción e identidad, (pp. 109-136). Estocolmo: Universidad de Estocolmo.

Boroditsky, L. (2001). Does language shape thought? English and Mandarin speakers' conceptions of time. Cognitive Psychology, 43,1.1-22.

Caffi, C. \& Janney, R. (1994). Toward a pragmatics of emotive communications. Journal of Pragmatics, 22, 325-373.

Chen, S., Kennedy, M. \& Zhou, Q. (2012). Parents'expression and discussion of emotion in the multilingual family: Does language matter? Perspectives on Psychological Science, 7(4), 365-383.

Dewaele, J. M. \& Pavlenko, A. (2002). Emotion vocabulary in interlanguage. Language Learning, 52(2), 263-322.

Eisenberg, N., Cumberland, A. \& Spinrad, T. (1998). Parental socialization of emotions. Psychological Inquiry, 9(4), 241-273.

Evans, N. \& Levinson, S. (2009). The myth of language universals. Language diversity and its importance for cognitive science. Behavioral and Brain Sciences, 32, 429-492.

Halliday, M. A. K. (1984). Introduction to functional grammar. London: Edward Arnold.

Harris, C., Berko Gleason, J. \& Ayçiçegi, A. (2006). When is first language more emotional? Psychoophysiological evidence from bilingual speakers. En Pavlenko, A. (ed.). Bilingual minds. Emotional experience, expresión and representation, (pp. 257-283). Clevedon, UK: Multilingual Matters.

Hoff, E., Core, C., Place, S., Rumiche, R., Señor, M. \& Parra, M. (2012). Dual language exposure and early bilingual development. Journal of Child Language, 39, 1-27.

Hoff, E. (2013). Language development. Belmont, CA: Wadsworth/Cengage Learning.

Hunston, S. \& Geoff, T. (comp.). (2001). Evaluation in text: Authorial stance and the construction of discourse. Oxford: Oxford University Press.

Kaplan, N. (2009). "Héroes, villanos y víctimas": La construcción discursiva de personajes en las noticias televisivas sobre eventos conflictivos. En M. Shiro, P. Bentivoglio \& F. Erlich (eds.). Haciendo discurso. Homenaje a Adriana Bolivar. Caracas: Universidad Central de Venezuela.

Koven, M. (2006). Feeling in two languages: A comparative analysis of a bilingual's affective displys in French and Portuguese. En A. Pavlenko (ed.) (2006). Bilingual minds. Emotional experience, expresión and representation, (pp. 84-117). Clevedon, UK: Multilingual Matters.

Labov, W. (1972). The transformation of experience in narrative syntax. En W. Labov (ed.). Language in the inner city: Studies in the Black English vernacular, (pp. 354-405). Philadelphia: University of Pennsylvania.

Lázaro Mora, F. (1999). La derivación apreciativa. En I. Bosque y V. Demonte. Gramática descriptiva de la lengua española, (pp. 4645-4682). Madrid: Espasa Calpe.

Lucy, J. \& Gaskins, S. (2001). Grammatical categories and the development ofclassification: a comparative approach. En M. Bowerman y S. Levinson (eds.). Language acquisition and conceptual development, (pp. 257-283). Cambridge: Cambridge University Press.

Martin, J. R. \& White, P. R. (2005). The language of evaluation: Appraisal in English. New York: Palgrave Macmillan.

MacWhinney, B. (2000). The CHILDES project: Tools for analyzing talk. Hillsdale, NJ: Lawrence Erlbaum.

Montrul, S. (2013). El bilingüismo en el mundo hispanohablante. Singapore: Wiley-Blackwell.

Oatley, K. \& Jenkins, J. (1996). Understanding emotions. Cambridge, MA: Blackwell.

Ochs, E. (1988). Culture and language development. Language acquisition and language socialization in a Samoan village. Cambridge: Cambridge University Press.

Panaiyotou, A. (2006). Translating guilt: An endeavor of shame in the Mediterranean? En Pavlenko, A. (ed.) (2006). Bilingual minds. Emotional experience, 
expression and representation, (pp. 183-208). Clevedon, UK: Multilingual Matters.

Pavlenko, A. (2005). Emotions and multilingualism. Cambridge: Cambridge University Press.

Pavlenko, A. (2006). Bilingualselves. En A. Pavlenko (ed.) (2006). Bilingual minds. Emotional experience, expression and representation, (pp. 1-33). Clevedon, UK: Multilingual Matters.

Pedraza, P. (1985). Language maintenance among New York Puerto Ricans. En L. Elías-Olivares (ed.), Spanish language use and public life in the United States, (pp. 59-71). New York: Mouton de Gruyter.

Pew Research Hispanic Center. (2013). Statistical portrait of the foreign-born population in the United States, 2011. Recuperado de: http://www. pewhispanic.org/2013/01/29/statisticalportrait-of-the-foreign-born-population-in-theunited-states-2011/

Quirk, R., Greenbaum, S., Leech, G. \& Svartvik, J. (1989). A comprehensive grammar of the English language. Londres: Longman.

Sarangi, S. (2003). Editorial. Evaluating evaluative language. Text: An interdisciplinary journal for the study of discourse, 20(2). 165-170.

Schiefflin, B. \& Ochs, E. (1986). Language socialization across cultures. Cambridge: Cambridge University Press.
Shiro, M. (1997). Labov's model of narrative analysis as an emerging study in discourse. Journal of Narrative and Life History, 7(1-4). 309-314.

Shiro, M. (2003). Genre and evaluation in narrative development. Journal of Child Language, 13, 217248.

Shiro, M. (2007). La construcción del punto de vista en los relatos orales de niños en edad escolar: un análisis discursivo de la modalidad. Caracas: Universidad Central de Venezuela.

Snow, C. (1995). Issues in the study of input: finetuning, universality, individual and developmental differences, and necessary causes. En P. Fletcher \& B. MacWhinney (eds.), The bandbook of child language, (pp. 180-193). Oxford: Blackwell.

Sokolov, J. \& Snow, C. (ed.). (1994). Handbook of research in language development using CHILDES. Hillsdale, NJ: Lawrence Erlbaum.

Urciuoli, B. (1996). Exposing prejudice: Puerto Rican experiences of language, race and class. Boulder, Colorado: Westview Press.

Verschueren, J. (1999). Understanding pragmatics. London: Arnold.

Wierzbicka, A. (1999). Emotions across languages and cultures. Diversity and universals. Cambridge: Cambridge University Press.

Recibido: 01 de mayo de 2013

Aceptado: 5 de setiembre de 2013

Actualidades en Psicología, 27(115), 2013, 75-91 